Research Article

\title{
Radar Working State Recognition Based on the Unsupervised and Incremental Method
}

\author{
Huiqin Li ${ }^{(D)}$, Yanling Li $\left(\mathbb{D}\right.$, Chuan He ${ }^{\mathbb{D}}$, Hui Zhang, and Jianwei Zhan
}

Xi'an Research Institute of High Technology, Xi'an 710025, China

Correspondence should be addressed to Yanling Li; lyling998@163.com

Received 26 July 2021; Revised 4 September 2021; Accepted 15 September 2021; Published 7 October 2021

Academic Editor: Tomasz Wandowski

Copyright (C) 2021 Huiqin Li et al. This is an open access article distributed under the Creative Commons Attribution License, which permits unrestricted use, distribution, and reproduction in any medium, provided the original work is properly cited.

\begin{abstract}
Radar working state recognition is the basis of cognitive electronic countermeasures. Aiming at the problem that the traditional supervised recognition technology is difficult to obtain prior information and process the incremental signal data stream, an unsupervised and incremental recognition method is proposed. This method is based on a backpropagation (BP) neural network to construct a recognition model. Firstly, the particle swarm optimization (PSO) algorithm is used to optimize the preference parameter and damping factor of affinity propagation (AP) clustering. Then, the PSO-AP algorithm is used to cluster unlabeled samples to obtain the best initial clustering results. The clustering results are input as training samples into the BP neural network to train the recognition model, which realizes the unsupervised recognition. Secondly, the incremental AP (IAP) algorithm based on the $K$-nearest neighbor (KNN) idea is used to divide the incremental samples by calculating the closeness between samples. The incremental samples are added to the BP recognition model as a new known state to complete the model update, which realizes incremental recognition. The simulation experiments on three types of radar data sets show that the recognition accuracy of the proposed model can reach more than $83 \%$, which verifies the feasibility and effectiveness of the method. In addition, compared with the AP algorithm and $K$-means algorithm, the improved AP method improves $59.4 \%, 17.6 \%$, and $53.5 \%$ in purity, rand index (RI), and F-measure indexes, respectively, and the running time is at least $34.8 \%$ shorter than the AP algorithm. The time of processing incremental data is greatly reduced, and the clustering efficiency is improved. Experimental results show that this method can quickly and accurately identify radar working state and play an important role in giving full play to the adaptability and timeliness of the cognitive electronic countermeasures.
\end{abstract}

\section{Introduction}

With the diversity of radar systems, the complexity of electromagnetic signals, the intelligence of the countermeasure targets, and the development application of new antijamming technologies, the combat effectiveness of traditional electronic countermeasure systems has gradually declined. There is an urgent need to transform from "artificialization" to "intelligence." The United States military first proposed the concept of "cognitive electronic warfare" in 2010. It successively released project announcements such as "Adaptive Electronic Warfare Behavioral Learning (BLADE)" and "Adaptive Radar Countermeasures (ARC)" [1]. Aiming at new multifunctional radars, a radar working state recognition model is built through the machine learning algorithm to infer the behavior intention of the system. Thus, the capability of real-time attack and adaptive to the environment is improved.

Radar working state recognition is the crucial link in cognitive electronic warfare. It specifically refers to acquiring radar signal characteristic parameters and using the data characteristics to explore signal parameter rules and identify its working state. The rapid and accurate recognition of the radar working state is essential for evaluating the radar threat level, distributing the jamming resources, and giving full play to the electronic combat effectiveness.

Radar working state recognition is essentially a pattern classification problem, so it can be solved using the classification algorithm in machine learning theory. At present, 
most scholars are mainly based on prior information using supervised learning methods such as Bayesian classifier [2, 3], decision tree $[4,5]$, and support vector machine $[6,7]$ to identify the working state. The BP neural network has strong selflearning and nonlinear mapping capabilities, which is particularly suitable for solving problems with complex internal mechanisms and can establish the mapping relationship between characteristic signal parameters and radar working conditions. It is the most widely used in working state recognition [8-11]. Still, there are two problems with the BP neural network in the virtual electronic countermeasure environment: (1) This method requires labeled sample data before classification. Still, noncooperative radar's signal characteristic parameters and corresponding working state are hard to get, so the problem of the lack of prior knowledge identification cannot be solved. (2) Above methods are off-line batch learning methods. Aiming at the incremental signal data stream generated in the engagement process, it cannot judge online whether it belongs to a new state and update the recognition model in time. Because the unsupervised learning method does not need sample labels, the clustering method can be used to complete sample prelabeling to build a knowledge base. The data in the knowledge base can be used as a training set and input into the BP neural network to improve the recognition accuracy. Therefore, to solve the problems of adaptability and timeliness of recognition, the research on the unsupervised incremental recognition method based on the combination of clustering and classification has more practical application significance.

Common unsupervised clustering algorithms include partition-based clustering, density-based clustering, and fuzzy-based clustering. In studies $[12,13]$, the $K$-means algorithm has been used to complete radar signal sorting and recognition. The process is simple and efficient. However, this algorithm needs to set the $K$ value in advance and is very sensitive to noise and outliers. Some studies $[14,15]$ have proposed an adaptive density clustering algorithm for radar signal features, which can cluster the arbitrary shape distribution. However, the clustering results are related to the algorithm parameters, and the parameter tuning process is complex. Other studies $[16,17]$ have introduced a radar signal classification method based on fuzzy $C$-means clustering, which overcomes the influence of noise and missing data. Still, the algorithm's performance depends on the initial clustering center. AP algorithm is a relatively new clustering algorithm [18], which does not have to predetermine cluster number and initial cluster centers. It can efficiently solve the problem of the non-Euler distribution of data. Therefore, it can be used to solve the problem of noncooperative target state recognition without prior information. However, the preference parameter and damping factor are two important parameters affecting the clustering algorithm's effects [19]. The former affects the number of clusters, while the latter affects the convergence speed and accuracy. Parameter values are usually based on experience. The process is complicated and difficult to obtain the optimal parameters. Moreover, when new training data arrives, the new data needs to be merged into the original data set and recluster based on the whole new data set [20]. Therefore, the recognition model cannot be updated in time and increases the space and time.
An unsupervised and incremental radar working state recognition method is proposed in this paper to solve the above problems. The innovations and main contributions include the following:

(1) An unsupervised and incremental recognition model is proposed, mainly divided into three steps: the initial sample prelabeling based on PSO-AP, the recognition model building based on BP, and the incremental sample clustering based on IAP

(2) The parameter optimization ability of the PSO algorithm [21] is used to obtain the best preference parameter and damping factor parameter of the AP algorithm, automatically eliminate the AP algorithm oscillation, converge to the optimal clustering result, and improve clustering accuracy

(3) The BP neural network recognition model is built. The clustering results of PSO-AP are used as a prior knowledge base to input into the recognition model and improve recognition accuracy

(4) The idea of incremental learning is introduced, and the IAP algorithm based on KNN is proposed. Based on the PSO-AP clustering results, samples are classified online by calculating the compactness between incremental samples and sample data in KNN instead of the whole samples. Thus, a lot of space and time costs are saved

(5) The three radars provided in studies [22, 23] are taken as the research objects. Simulation experiments show that the recognition algorithm combining IAP clustering with PSO and BP neural network improves the recognition accuracy and efficiency. This method achieves good classification and recognition for known and unknown states, which proves the feasibility and effectiveness of the proposed method

The rest of this paper is organized as follows. The radar working state recognition model based on the unsupervised and incremental method is presented in Section 2. A detailed introduction to the key algorithms of the recognition model is presented in Section 3. The simulation experiment and analysis of the results are presented in Section 4. Finally, the conclusions drawn from this study are discussed in Section 5.

\section{Radar Working State Recognition Based on the Unsupervised and Incremental Method}

2.1. Radar Working State Recognition Process. Due to the military sensitivity and complexity of radar signals, it is challenging to obtain real-time signal parameters and greatly increase the difficulty of radar working state recognition. Therefore, this paper proposes the radar working state recognition process, as shown in Figure 1. The process is divided into four steps: Firstly, the signal features that identify the radar working state are extracted. Secondly, the data set is randomly generated according to the range of characteristic signal parameters. Then, the data set is preprocessed. Finally, the data is input into 


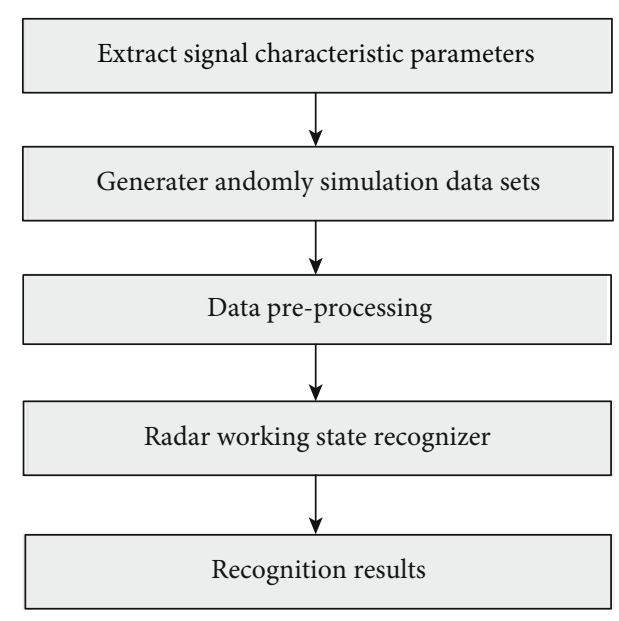

FIGURE 1: Radar working state recognition process.

the radar working state recognizer, and the recognition result is output.

(1) Extraction of signal characteristic parameters: the radar working state characteristic parameters include replay frequency, pulse width, duty cycle, and carrier frequency. When the radar is in different working states, the signal's characteristic parameters also have significant differences

(2) Data set simulation: it is difficult to obtain the measured radar data due to the constraint of experimental environment conditions. According to the radar state characteristic parameter range table, samples for each state are randomly generated to construct the simulation experiment data set

(3) Data preprocessing: data preprocessing mainly normalizes the data set to eliminate the influence of parameters' dimensional difference and improve the recognition accuracy. After normalization, according to the two cases of radar known and unknown working state recognition, the data set is randomly divided into initial samples and incremental samples by proportion

(4) Radar working state recognizer: the function of the recognizer is to output the corresponding radar working state recognition results according to the input signal characteristic parameters. The recognizer adopted in this paper is based on the unsupervised and incremental recognition method to realize the radar working state recognition

2.2. Recognition Model Based on the Unsupervised and Incremental Method. To quickly and accurately complete the prelabeling of initial samples without prior information and the online division of incremental samples, achieve the known and unknown working state recognition of incremental signal samples, and further improve cognitive electronic countermeasure system efficiency, this paper uses label samples generated by improved AP clustering as a training set of the BP model. It generates an unsupervised and incremental radar working state recognition model. The model's step flow is shown in Figure 2.

The specific steps are as follows:

Step 1. The data set is generated randomly, and the input parameters are normalized to make it in $[0,1]$. The data set is divided into initial samples and incremental samples.

Step 2. PSO-AP algorithm is used to prelabel the initial sample data to get the training samples with labels, and the number of samples in the clustering center and each cluster is recorded.

Step 3. Input AP initial clustering results into BP neural network as prior knowledge to build the recognition model.

Step 4. Adopt the KNN idea to IAP clustering for incremental samples. If a known state is judged, then return to Step 3 to complete recognition of the known state. If it is unknown, then update the clustering results and perform Step 5.

Step 5. When the incremental sample reaches a certain size, the clustering cluster is input into the BP neural network as a new training sample. The recognition model is updated to complete the unknown state detection. Otherwise, return to Step 4.

\section{Algorithm Analysis}

\subsection{PSO-AP Algorithm}

3.1.1. AP Algorithm. AP algorithm is a new clustering algorithm based on "information transfer" between data points, proposed in Science magazine in 2007. Unlike other clustering algorithms such as $K$-means, AP does not need to set the number and center of clustering before clustering, which is widely used in face classification and image segmentation. The AP clustering process is shown in Figure 3. The principle is to regard all the data points as potential clustering center, and the connection lines between data points form a network, that is, similarity matrix. Then, constantly update and pass the availability and responsibility information between points through similarity matrix, and calculate the cluster center of each sample for automatic clustering.

The algorithm can be summarized as the following steps:

Step 1. According to equation (1), the Euclidean distance is used to calculate the similarity between data points $i$ and points $j$, and the similarity matrix $s(i, j)$ is obtained. Elements on the diagonal are defined as the preference $p(i)$.

$$
s(i, j)= \begin{cases}-\left\|x_{i}-x_{j}\right\|^{2}, & i \neq j, \\ p, & i=j,\end{cases}
$$

where the smaller the $s(i, j)$ is, the lower the ability of the point $j$ as the center of point $i$ clustering is.

Step 2. Set $r=0$ and $a=0$. Calculate the availability and responsibility according to

$$
r(i, j)= \begin{cases}s(i, j)-\max _{j^{\prime} \neq j}\left\{s\left(i, j^{\prime}\right)\right\}, & i=j, \\ s(i, j)-\max _{j^{\prime} \neq j}\left\{a\left(i, j^{\prime}\right)+s\left(i, j^{\prime}\right)\right\}, & i \neq j,\end{cases}
$$




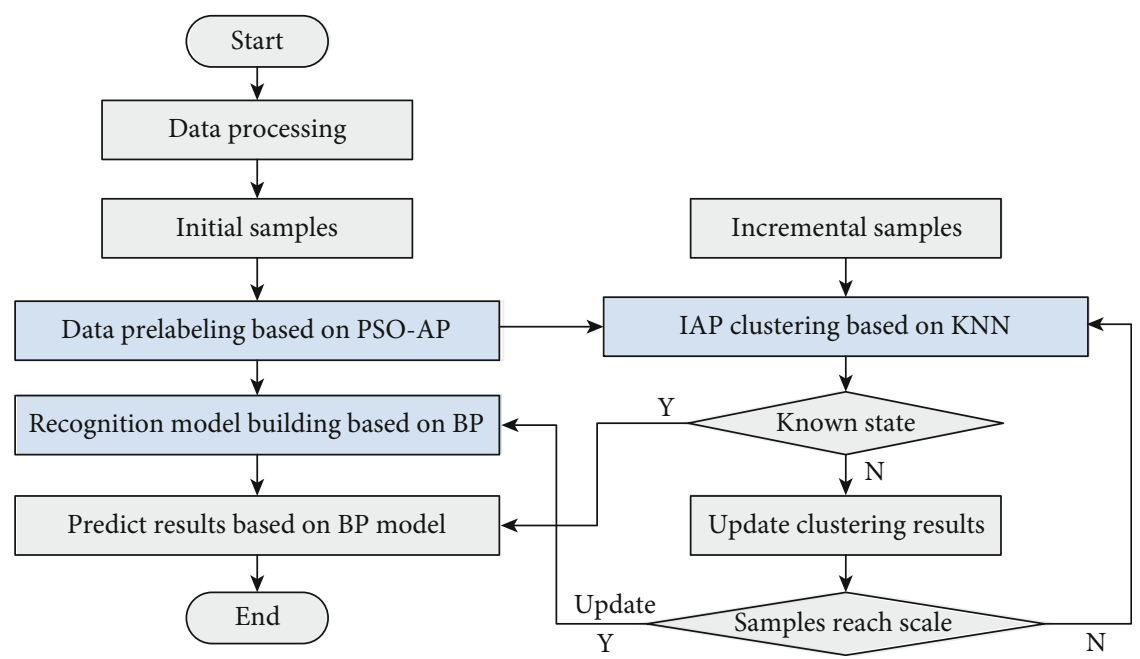

FIGURE 2: Recognition model based on the unsupervised and incremental method.
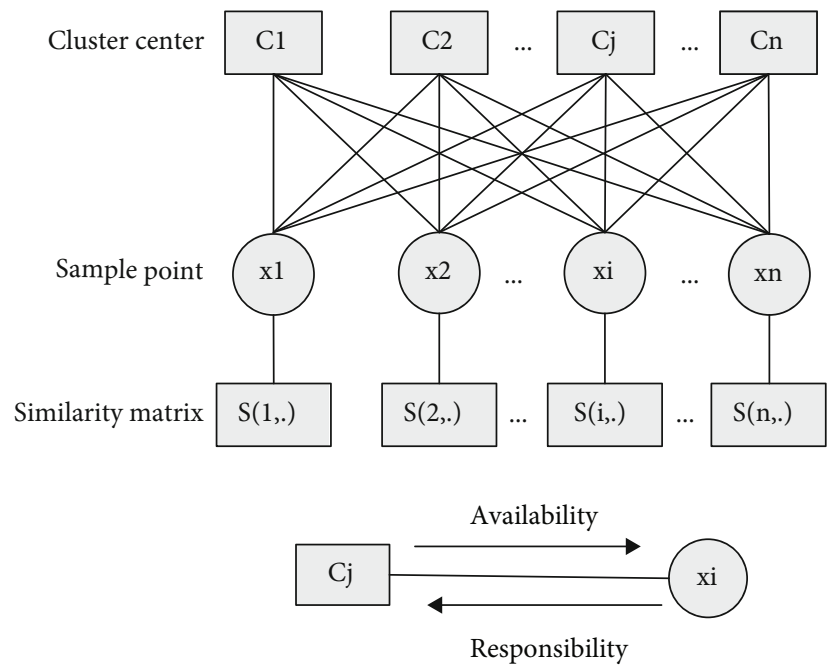

FIGURE 3: Clustering process of AP algorithm.

$a(i, j)= \begin{cases}\left.\sum_{i^{\prime} \neq j} \max \left\{0, r\left(i^{\prime}, j\right)\right\}\right\}, & i=j, \\ \min \left\{0, r(j, j)+\sum_{i^{\prime} \neq i, j} \max \left\{0, r\left(i^{\prime}, j\right)\right\}\right\}, & i \neq j,\end{cases}$

where $r(i, j)$ represents the degree to which the point $j$ is suitable for the clustering center of the point $i$ and $a(i, j)$ represents the degree to which the point $i$ selects the point $j$ as its clustering center.

Step 3. To avoid the oscillation of the AP algorithm in the iterative process, a damping factor $\lambda$ is added in each iteration, and the information transmission process is updated as follows:

$$
\begin{aligned}
& r_{t+1}=(1-\lambda) r_{t+1}+\lambda r_{t}, \\
& a_{t+1}=(1-\lambda) a_{t+1}+\lambda a_{t} .
\end{aligned}
$$

Step 4. Determine the cluster center point $k$ according to

$$
k=\operatorname{argmax}\{a(i, k)+r(i, k)\} .
$$

Step 5. Iterate and update $r$ and $a$. When the maximum number of iterations is reached, or the clustering center remains unchanged after multiple iterations, the iteration is stopped, and the remaining data objects are allocated to the corresponding clustering. Otherwise, continue with Step 2-Step 4.

3.1.2. AP Algorithm Parameter Optimization Based on PSO. The clustering efficiency of the AP algorithm is mainly affected by preference $p$ and damping factor $\lambda$. Among them, the larger the $p$ is, the greater the clustering number is. The $p$ is generally the mean of the similarity matrix [18]. However, it cannot always obtain the optimal clustering result due to the difference in data sets. The smaller the $\lambda$ is, the greater the change of $r$ and $a$ is, and the number of iterations will be reduced. In general, $\lambda$ is debugged in $(0,1)$. However, the process is complex, and it is difficult to obtain the best parameters. As a swarm intelligence optimization algorithm, PSO finds the optimal solution through the simple behavior of personal particles and the information interaction with the global particles. It has the characteristics of simple operation, fast convergence, and strong global searchability. In recent years, scholars have increasingly applied it to data processing and proposed some new PSO methods. For example, facing the challenge of the "curse of dimensionality" when dealing with high-dimensional data, Song et al. [24] proposed a variable-size cooperative coevolutionary PSO algorithm for feature selection (FS). Focusing on the FS problem with fuzzy cost, Hu et al. [25] proposed a fuzzy multiobjective FS method with PSO. Xue et al. [26] proposed an adaptive PSO algorithm to solve the local optimal stagnation in large-scale FS. In this 
paper, we use PSO to optimize the parameters of AP clustering. The specific implementation steps of the PSO-AP algorithm are as follows:

Step 1. $p$ is in $[1 . \overline{5} s, 0.1 \bar{s}]$, where $\bar{s}$ is the mean of the similarity matrix. $\lambda$ is in $[0.5,0.95]$. Initialize the particle swarm parameters. Set the population size $N$ and maximum number of iterations $T$. This paper selects purity as the particle fitness function. Purity is one of the clustering evaluation validation indexes, and its overall idea is to divide the correct number of samples by the total number of samples. The calculation formula is

$$
\text { fitness }=\operatorname{purity}(\Omega, C)=\frac{1}{N} \sum_{k} \max _{j}\left|\omega_{k} \cap c_{j}\right|,
$$

where $N$ is the total number of samples, $\Omega=\left\{\omega_{1}, \omega_{2}, \cdots\right.$ ,$\left.\omega_{k}\right\}$ is the sample set of clustering, $\omega_{k}$ is the clustering cluster $k$ th, $C=\left\{c_{1}, c_{2}, \cdots, c_{j}\right\}$ is the sample set, and $c_{j}$ is the sample $j$ th.

Study [27] mentioned that the Silhouette (clustering evaluation index) is taken as the fitness function. Because the Silhouette introduces distance calculation, and different data sets are suitable for different distance calculation rules, it is difficult to calculate the Silhouette in a unified way. Moreover, purity is the evaluation index with label, and the Silhouette is the evaluation index without label. Many experiments show that the evaluation index with label is better than that without label, so it is more appropriate to take purity as the fitness function.

Step 2. The particles' dimension $D$ is determined by $p$ and $\lambda$, and the position and velocity are initialized randomly.

Step 3. Calculate fitness value, personal extremum, and global extremum according to equations (1)-(6).

Step 4. In each iteration, a particle updates its position by learning the optimal position found by the particle itself, that is, the personal leader (Pbest) and the particle's neighborhoods, that is, the global leader (Gbest). Without loss of generality, assuming that the personal and global leaders of the $i$ th particle $X_{i}=\left(x_{i, 1}, \cdots, x_{i, D}\right)$ are $P b_{i}=\left(p b_{i, 1}, \cdots, p b_{i, D}\right)$ and $G b_{i}=\left(g b_{i, 1}, \cdots, g b_{i, D}\right)$, respectively, this particle is updated by using equations (7) and (8) [28].

$$
\begin{gathered}
v_{i, j}^{t+1}=\omega v_{i, j}^{t}+c_{1} r_{1}\left(p b_{i, j}^{t}-x_{i, j}^{t}\right)+c_{2} r_{2}\left(g b_{i, j}^{t}-x_{i, j}^{t}\right), \\
x_{i, j}^{t+1}=x_{i, j}^{t}+v_{i, j}^{t+1},
\end{gathered}
$$

where $i \in N, j \in D, r_{1}$, and $r_{2}$ are the random numbers between $[0,1], t$ is the current iteration number, $\omega$ is the inertia factor, and $c_{1}$ and $c_{2}$ are the learning factors.

Step 5. Update the particle swarm fitness value, individual extremum, and population extremum, and regard the particle with the largest fitness value in each generation as the current optimal particle.

Step 6. When the maximum number of particle swarm iteration is reached, the optimal values are output as the optimal $p$ and $\lambda$. Otherwise, repeat Step 2-Step 5.

Step 7. Substitute the optimal $p$ and $\lambda$ into the AP algorithm to complete clustering.
3.2. BP Algorithm. The BP neural network's basic idea is to realize the nonlinear mapping relationship between input samples and output samples using the forward propagation of signals and the backpropagation of errors. Figure 4 shows a typical structure of a three-layer BP neural network model. The sample characteristic signal is firstly transmitted from the input layer to the hidden layer and then reaches the output layer after calculation. Next, the output result is calculated as the radar working state. When the actual output result is inconsistent with the expected output result, the error will be transmitted back. The weight of each element will be corrected by the gradient descent method. Repeat the sample until the network output error is reduced to an acceptable level or reaches a default number of learning times.

3.3. IAP Algorithm Based on KNN. In cognitive electronic warfare, the jammer usually receives the new signal samples in batches or streams. The traditional AP algorithm needs to combine the new training data into the original data set to retrain the whole model, lacking adaptability and timeliness. The KNN algorithm is based on the idea that the unknown sample should be consistent with most known samples in its neighbors. It divides the incremental data by calculating the $K$-nearest neighbors of the unknown sample. It has the characteristics of mature theory, low time complexity, and incremental learning ability and can achieve efficient incremental clustering combined with the AP algorithm. To complete the online recognition of the unknown signal sample, this paper proposes an IAP algorithm with the idea of KNN. The algorithm is based on the existing AP clustering results to update the model with the new sample data and improve the clustering efficiency. The incremental processing process is shown in Figures 5 and 6.

The specific steps of the algorithm are as follows:

Step 1. The PSO-AP algorithm is used to cluster the samples to obtain the clustering center and the number of samples in each cluster.

Step 2. Calculate the distance between the incremental sample and the initial sample, use the cross-validation method to determine the $K$ value of $\mathrm{KNN}$, and get the cluster to which each nearest neighbor belongs.

Step 3. Calculate the average distance $D$ between nearest neighbors, and calculate the average distance $d_{1}, d_{2}, \cdots, d_{n}$ between incremental data and each cluster in the nearby neighbors, where $n$ is the number of clusters.

Step 4. If $D>\min \left\{d_{1}, d_{2}, \cdots, d_{n}\right\}$, then the increment belongs to the cluster of $\min \left\{d_{1}, d_{2}, \cdots, d_{n}\right\}$. Otherwise, it belongs to the new cluster.

Step 5. When the incremental data scale is reached, stop incremental clustering. Otherwise, loop through Steps 2-4.

\section{Simulation Experiment and Analysis of Results}

The experimental simulation platform of this paper is as follows:

(i) The operating system is windows 10 


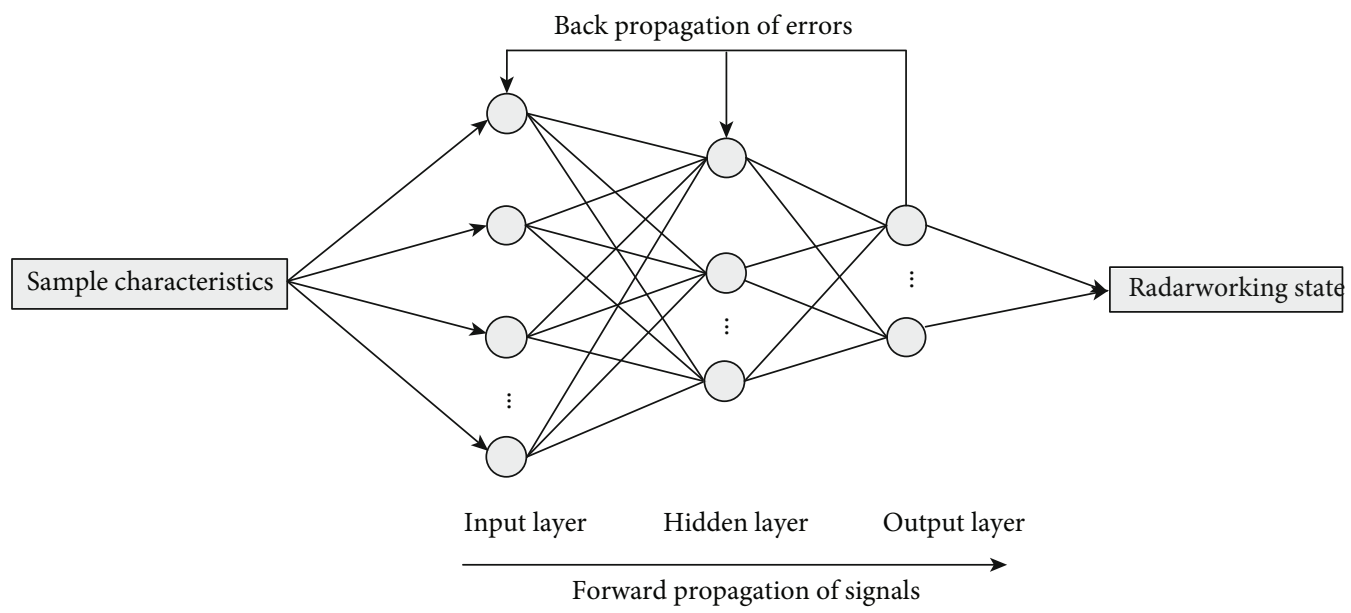

Figure 4: BP neural network model.

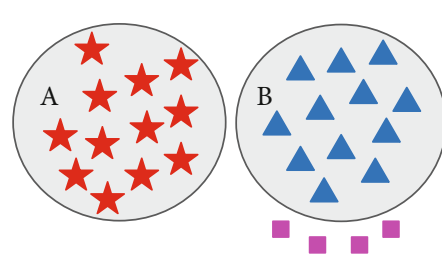

C

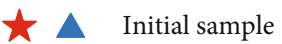

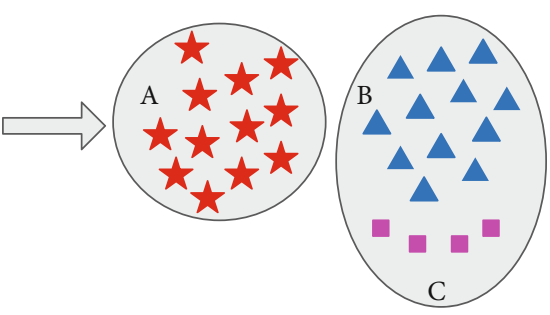

- Incremental sample

Figure 5: Add to an existing cluster.

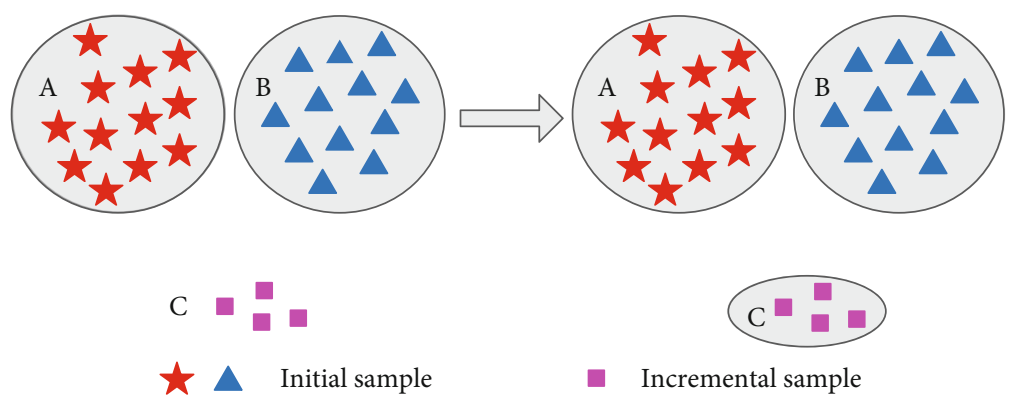

FIgURE 6: Create a new cluster.

(ii) The CPU is Intel(R) Core (TM) i7 $2.6 \mathrm{GHz}$

(iii) The memory is $8 \mathrm{~GB}$

(iv) The programming tool is MATLAB R2016b

The algorithm is verified, and the experimental results are processed by the MATLAB R2016b tool. Taking the airborne phased array radar (APAR) in the air-air combat mode in reference [22] and the radar $\mathrm{A}$ and $\mathrm{B}$ provided in reference [23] as examples, three experiments were designed to verify the performance of the model algorithm: (1) validation of PSO-AP algorithm, (2) $K$ value optimization of IAP clustering, and (3) validation of radar known and unknown working state recognition.
4.1. Simulation and Preprocessing of Data Sets. Due to the military confidentiality of the radar, the actual signal parameters of the radar working state are difficult to obtain. Many studies $[22,23]$ generate data randomly based on the working parameter ranges. Therefore, according to the parameter range in Tables 1 and 2, this paper randomly generated 100 samples for each state to form a data matrix of $400 * 3$, $400 * 3$, and $600 * 5$ dimensions, respectively. The simulation data set is shown in Table 3 .

This paper uses the improved AP algorithm to analyse signal characteristic parameters, and the AP algorithm is based on the similarity between the data points for clustering. If the data is an order of magnitude difference, the error will be great when similarity is obtained by using the Euclidean distance. 
TABLE 1: Radar A and B working parameter ranges [22].

\begin{tabular}{lcccc}
\hline $\begin{array}{l}\text { Radar } \\
\text { type }\end{array}$ & State & $\begin{array}{c}\text { Refrequency } \\
(\mathrm{GHz})\end{array}$ & $\begin{array}{c}\text { Pulse reinterval } \\
(\mu \mathrm{s})\end{array}$ & $\begin{array}{c}\text { Pulse width } \\
(\mu \mathrm{s})\end{array}$ \\
\hline \multirow{4}{*}{ A } & S1 & $9.7-9.9$ & $3-10$ & $1-3$ \\
& S2 & $9.7-9.9$ & $3-125$ & $0.1-20$ \\
& S3 & $9.5-9.8$ & $50-100$ & $0.1-0.5$ \\
& S4 & $9.5-9.8$ & $50-500$ & $1-200$ \\
\hline \multirow{3}{*}{ B } & S1 & $9.15-9.45$ & $500-2000$ & $1-200$ \\
& S2 & $9.15-9.45$ & $50-500$ & $0.1-10$ \\
& S3 & $8.5-10.6$ & $125-1000$ & $0.1-10$ \\
& S4 & $8.5-10.6$ & $100-1000$ & $3-60$ \\
\hline
\end{tabular}

TABLE 2: Radar APAR working parameter ranges [23].

\begin{tabular}{|c|c|c|c|c|c|}
\hline State & $\begin{array}{l}\text { Refrequency } \\
\quad(\mathrm{KHz})\end{array}$ & $\begin{array}{l}\text { Pulse } \\
\text { width } \\
\text { (ms) }\end{array}$ & $\begin{array}{l}\text { Duty } \\
\text { ratio } \\
(\%) \\
\end{array}$ & $\begin{array}{l}\text { Pulse } \\
\text { pressure } \\
\text { ratio }\end{array}$ & $\begin{array}{c}\text { Instantaneous } \\
\text { bandwidth } \\
(\mathrm{MHz})\end{array}$ \\
\hline S1 & $2-20$ & $1-200$ & $0.1-10$ & $1-16384$ & $0.2-100$ \\
\hline S2 & $2-300$ & $0.1-60$ & $\begin{array}{l}0.01- \\
50\end{array}$ & $1-16384$ & $0.2-500$ \\
\hline S3 & $0.5-5$ & $1-50$ & $1-10$ & $1-13$ & $0.1-1$ \\
\hline S4 & $0-20$ & $0.1-0.5$ & $0.1-1$ & $1-5$ & $1-10$ \\
\hline S5 & $10-300$ & $1-20$ & $1-33$ & $1-16$ & $0.1-1$ \\
\hline S6 & $6-20$ & $1-20$ & $1-25$ & $5-526$ & $1-10$ \\
\hline
\end{tabular}

TABLE 3: Radar simulation data set.

\begin{tabular}{lccc}
\hline Data set & Class number & Dimensionality & Sample number \\
\hline A & 4 & 3 & 400 \\
B & 4 & 3 & 400 \\
APAR & 6 & 5 & 600 \\
\hline
\end{tabular}

Therefore, the data needs to be normalized processing to improve the clustering effect of the AP algorithm. The commonly used normalization methods include deviation normalization and $Z$-score normalization. However, the former is affected by the maximum and minimum sample, leading to unstable normalization results, while the latter requires the distribution of data to be approximately Gaussian. To effectively narrow the gap between the data, the nonlinear normalization method was adopted in this paper, and the calculation formula is

$$
x^{\prime}=\arctan (x) *\left(\frac{2}{\pi}\right),
$$
data $x$.

where $x^{\prime}$ is the sample data after processing the original

4.2. Algorithm Evaluation Index. In this paper, common clustering validation indexes (such as purity, RI, and $F$-measure) and classification effect evaluation indexes (such as confusion matrix and accuracy) were selected to conduct quantitative analysis on the experimental results of the improved AP algorithm and BP model, respectively. Among them, the
TABLE 4: Confusion matrix.

\begin{tabular}{lcccc}
\hline \multirow{2}{*}{ Confusion matrix } & & \multicolumn{3}{c}{ Predicted states } \\
& & Class 1 & $\cdots$ & Class $N$ \\
\hline \multirow{3}{*}{ Actual states } & Class 1 & $\mathrm{a}$ & $\cdots$ & $\mathrm{b}$ \\
& $\ldots$ & $\ldots$ & $\ldots$ & $\ldots$ \\
& Class $N$ & $\mathrm{c}$ & $\ldots$ & $\mathrm{d}$ \\
\hline
\end{tabular}

calculation formula of purity is shown in equation (6), and the calculation methods of other indexes are shown in equations (10)-(12). The above quantitative evaluation indicators are all positive indicators.

4.2.1. RI.

$$
\mathrm{RI}=\frac{\mathrm{TP}+\mathrm{FN}}{\mathrm{TP}+\mathrm{FP}+\mathrm{FN}+\mathrm{TN}},
$$

where TP is the number of two similar sample points in the same cluster, FP is the number of two nonsimilar sample points in the same cluster, TN is the number of two nonsimilar sample points in different clusters, and FN is the number of two similar sample points in different clusters.

\subsubsection{F-Measure.}

$$
F=\frac{\left(\alpha^{2}+1\right) P * R}{\alpha^{2} *(P+R)},
$$

where $P=\mathrm{TP} /(\mathrm{TP}+\mathrm{FP}), \quad R=\mathrm{TP} /(\mathrm{TP}+\mathrm{FN})$, and the general default parameter $a=1$.

4.2.3. Confusion Matrix. A confusion matrix is a table for evaluating a classification model's performance, with rows representing actual values and columns representing predicted values. The confusion matrix's each unit represents the statistics corresponding to different predicted states and actual states, as shown in Table 4.

\subsubsection{Accuracy (ACC).}

$$
\mathrm{ACC}=\frac{\sum_{m=1}^{N} s_{m}}{k},
$$

where $k$ is the number of samples, $N$ is the number of sample classes, and $s$ is the number of correctly identified samples of class $m$ th.

\subsection{Experimental Results and Analysis}

\subsubsection{Validation of PSO-AP Algorithm}

(1) Algorithm Parameter Setting. In this experiment, the AP clustering purity was selected as the fitness function, and the preference and damping factor were selected as the particle dimensions. The maximum number of iterations of the AP clustering algorithm was 100. According to the commonly used range of other parameters, the PSO-AP algorithm's parameters are set as presented in Table 5. 
TABLe 5: Parameter settings of PSO algorithm.

\begin{tabular}{lcccccc}
\hline Population size & Iterations & Particle dimension & Speed range & Position scope & Learning factor & Inertia weight \\
\hline 20 & 50 & 2 & {$[-1,1]$} & {$[-5,5]$} & 2 & 1 \\
\hline
\end{tabular}

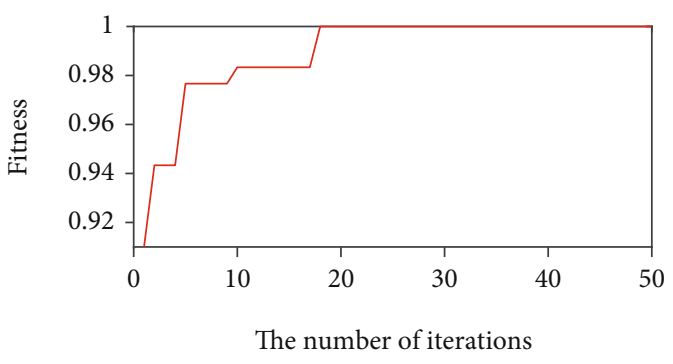

Figure 7: The relation curve between fitness and the number of iterations.

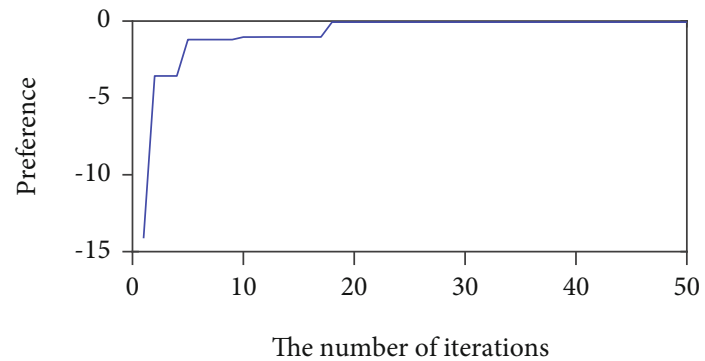

FIGURE 8: The relation curve between preference and the number of iterations.

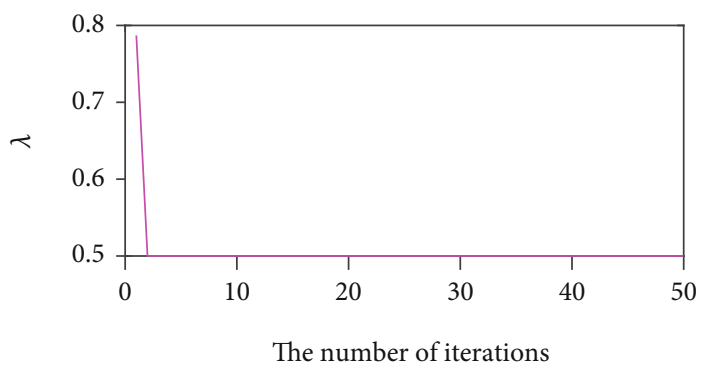

FIgURE 9: The relation curve between damping factor and the number of iterations.

(2) Result Analysis. Taking the radar APAR data set as an example, Figures 7-9 show the relationship among particle fitness value, preference, damping factor, and iteration number. As can be seen from the figures, the maximum number of particle iterations is 50 . When the iteration reaches the 18th generation, the fitness function tends to converge. At this point, the fitness is maximum, and the preference and damping factor are optimal. The values are as follows: fitness $=0.992, p=-0.0686$, and $\lambda=0.5$.

To compare and analyse algorithms, this experiment selected PSO-AP and AP algorithms to carry out clustering tests on three data sets, respectively. To avoid the randomness of the algorithms, a maximum value and a minimum value
TABle 6: Comparison of clustering indexes before and after optimization.

\begin{tabular}{lcccc}
\hline Data set & Algorithm & Purity & RI & $F$-measure \\
\hline \multirow{2}{*}{ A } & PSO-AP & 0.890 & 0.904 & 0.725 \\
& AP & 0.715 & 0.795 & 0.528 \\
\hline \multirow{2}{*}{ B } & PSO-AP & 0.805 & 0.848 & 0.599 \\
& AP & 0.661 & 0.782 & 0.424 \\
\hline \multirow{2}{*}{ APAR } & PSO-AP & 0.998 & 0.999 & 0.995 \\
& AP & 0.892 & 0.935 & 0.776 \\
\hline
\end{tabular}

were removed after running each algorithm 20 times and then took the average value. The results are shown in Table 6.

The comparison and analysis of purity, RI, and F-measure show that the PSO-AP algorithm has a better clustering effect on the three data sets than the AP algorithm. Purity, RI, and $F$ -measure indicators have increased by at least $11.9 \%, 6.8 \%$, and $41.3 \%$, respectively. The PSO-AP algorithm can obtain a better clustering effect because the PSO algorithm's good global search optimization ability is used to adjust the AP algorithm's parameters automatically. Firstly, the PSO algorithm rapidly obtains the optimal preference parameters and damping factors through continuously updating iterative optimization calculation. Then, according to the optimal preference parameters and damping factors, the AP algorithm completes clustering to obtain the best clustering effect. The PSO-AP algorithm can solve the setting value problem of the preference parameter and damping factor in the original AP algorithm and improve the clustering effect of the algorithm. Therefore, it is feasible to apply the PSO algorithm to the parameter optimization of the AP algorithm.

4.3.2. K Value Optimization of IAP Clustering. The key of the IAP algorithm based on KNN is the selection of $K$ value. If the $K$ value is too small, the model is too complex and prone to overfitting. If the $K$ value is too large, it is easy to cause fuzzy clustering. According to the rule of thumb, the $K$ value is usually odd and does not exceed the square root of the training sample. Considering the size of the sample data set in this paper, the range of $K$ values was set to all odd numbers between 1 and 19 .

In this experiment, according to the change law between the verification set's accuracy and the $K$ value, the 10 -fold cross-validation method was used to select the algorithm's optimal $K$ value. The specific method was to randomly divide the data set into ten groups of disjoint subsets. In each experiment, one group was successively selected as the verification set and the other nine groups as the training set. The verification set was taken as the incremental set. The average accuracy obtained through 10 times of IAP clustering experiment was used as the estimation of the algorithm accuracy, 


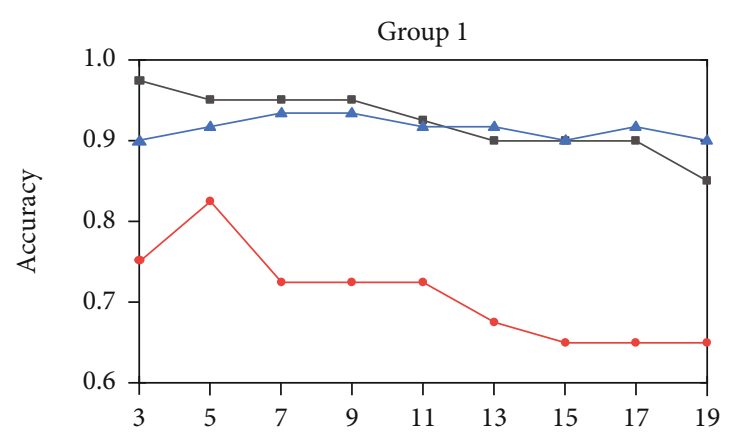

Value of K

(a)

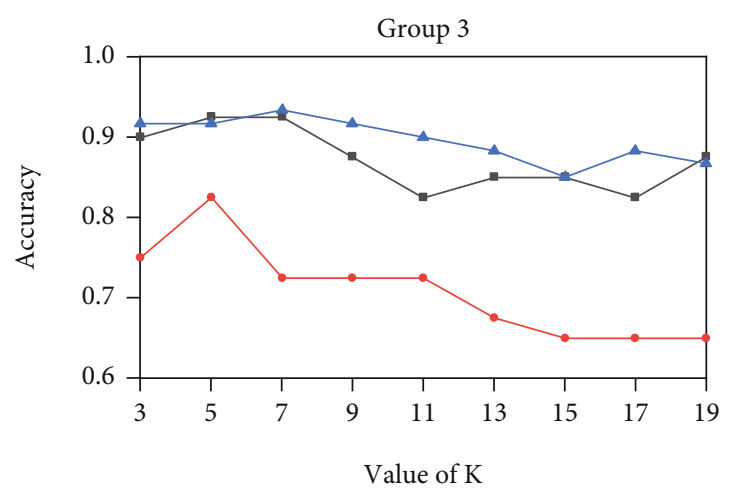

(c)

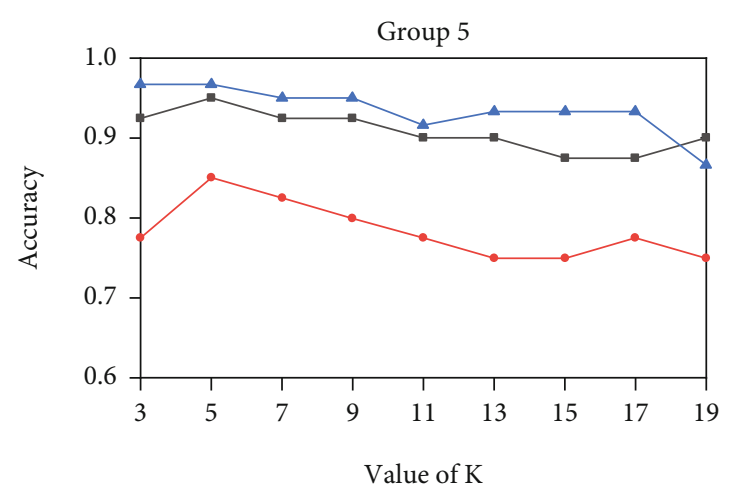

(e)

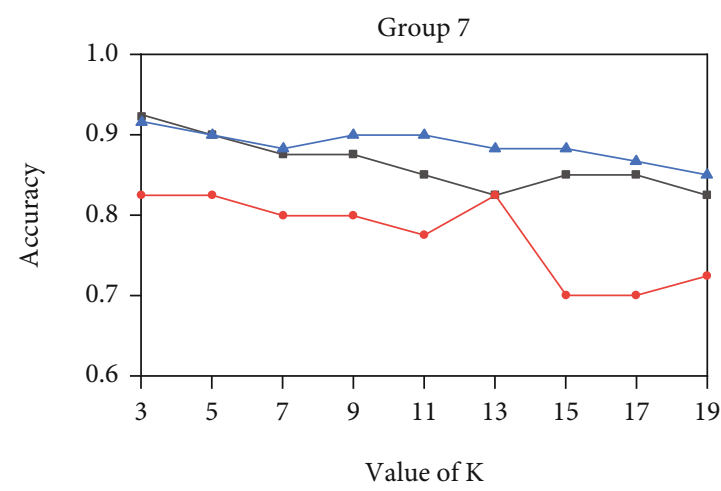

(g)

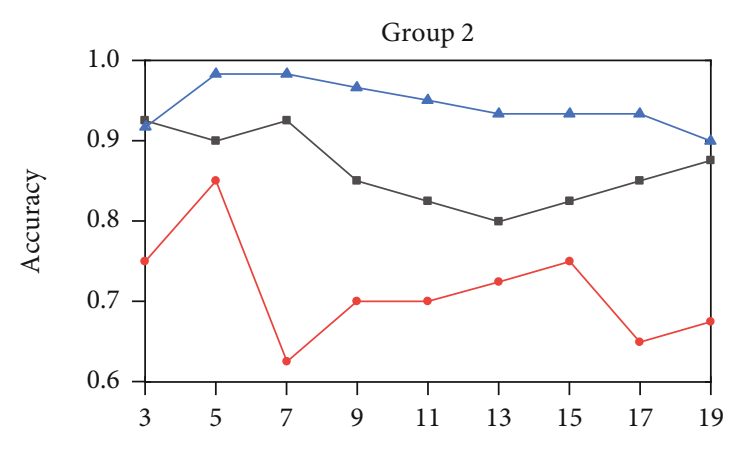

Value of K

(b)

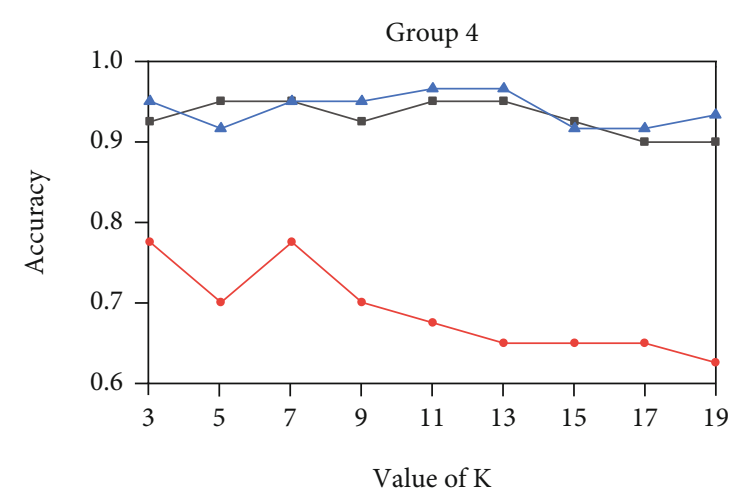

(d)

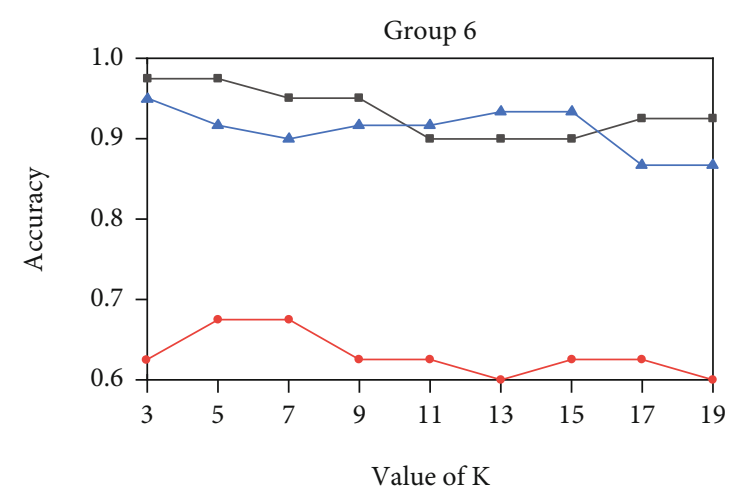

(f)

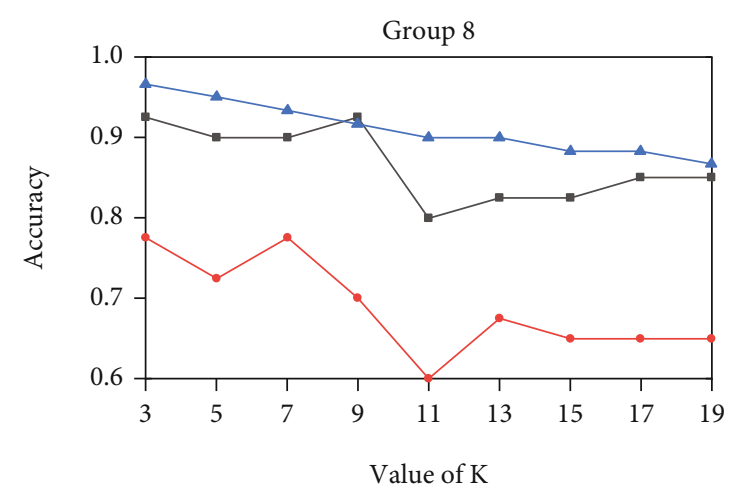

(h)

Figure 10: Continued. 


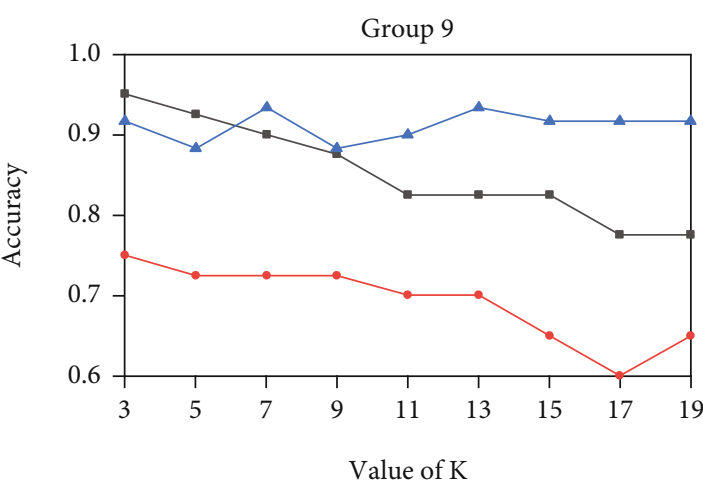

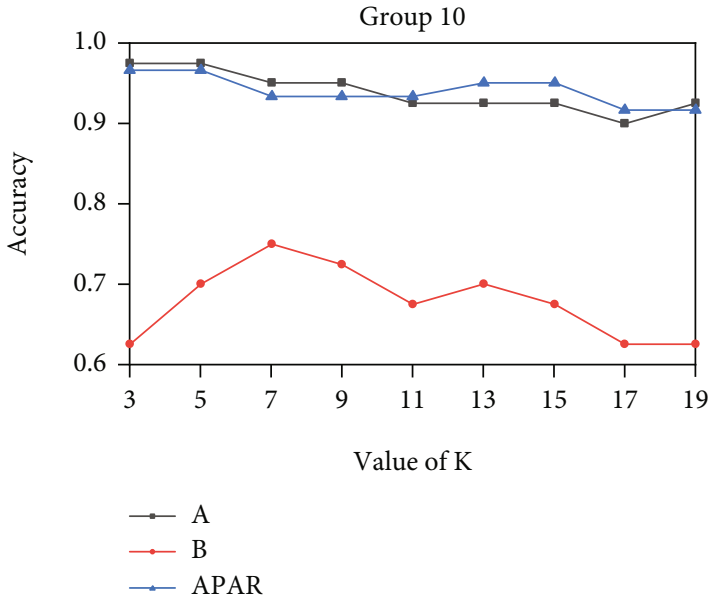

(j)

Figure 10: Relation curve between the accuracy of each incremental set and the change of $K$ value.

and the accuracy of all $K$ values was finally compared. The classification accuracy of each validation with the changing of $K$ value is shown in Figure 10.

Taking group 1 as an example, it can be seen from the figure that under this kind of grouping, when $K=3$ for the A data set, the classification accuracy of the verification set is the highest, which is $97.5 \%$. When $K=5$ for the B data set, the classification accuracy of the verification set is the highest, which is $82.5 \%$. When $K=7$ or $K=9$ for the APAR data set, the classification accuracy of the verification set is the highest, which is $93.33 \%$. Similarly, for other groups, the $K$ value at the highest accuracy of the verification set was selected as the best $K$ value in the current group. The accuracy of verification set at the corresponding value was statistically analysed. The statistical results show that the average classification accuracy of A, B, and APAR data sets is, respectively, $94.75 \%, 79 \%$, and $95 \%$.

To observe the influence of the $K$ value on the classification accuracy, the accuracy of the verification set with the same $K$ value under different groups was counted and averaged. The curve of the variation rule is shown in Figure 11. When the average accuracy of the verification set is the highest, the $K$ value was taken as the optimal value. As can be seen from the figure, A data set's optimal $K$ value is 3, B data set's optimal $K$ value is 5 , and APAR data set's optimal $K$ value is 7 . The $K$ value in the KNN has a great influence on the clustering result. When the $K$ value is set improperly, the clustering effect will be poor.

\subsubsection{Validation of Radar Known and Unknown Working State Recognition}

(1) Data Set Division. To verify the effectiveness of the radar working state recognition model proposed in this paper for known working state recognition and unknown status detection, the original data set constructed in Section 4.1 was divided into two types of data sets in this experiment:
(1) Assuming that all working states of the radar are known, $20 \%$ of the data from each class in the original data set were randomly selected as the incremental set and the remaining as the training set. The data division is shown in Table 7

(2) Assuming that a working state of the radar is unknown, a class in the original data set was taken as the incremental set of the unknown state and the other classes as the training set of the known state. $20 \%$ of the data from each class were randomly selected to form a test set for the experiment. The data division is shown in Table 8

(2) Effectiveness Analysis of IAP Clustering. Firstly, the training set and the incremental set were used to conduct the IAP clustering analysis for the known and unknown states, respectively. AP parameters were set using the method described in Section 4.3.1, and $K$ values of the data set were set using the method described in Section 4.3.2. Purity, RI, F-measure, and time were selected to measure the clustering effect. To facilitate the comparative analysis of the model, in this experiment, AP and $K$-means [29] algorithms are selected to verify the effectiveness of the IAP algorithm. The clustering number $k$ of the $K$-means algorithm is set as shown in Table 9.

The IAP, AP, and $K$-means algorithms were repeatedly run 20 times on A, B, and APAR data sets, respectively. After removing one maximum and one minimum value, take the average as the result. The clustering results are shown in Table 10 .

According to the comparison of simulation indicators of purity, RI, F-measure, and time, the following conclusions are drawn:

(1) From the perspectives of purity, RI, and F-measure, for the known and unknown working states of the three radar data sets, the clustering effect of the $K$ 


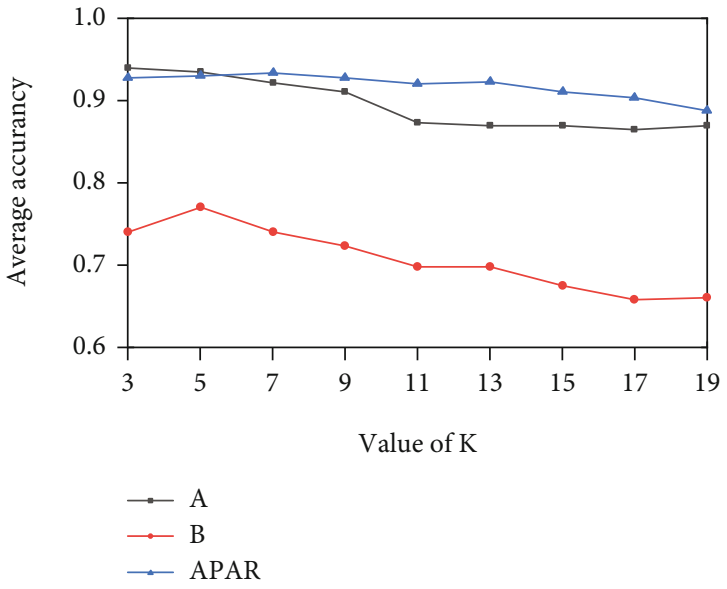

Figure 11: Relation curve between the average accuracy of each incremental set and the change of $K$ value.

TABLE 7: Division of known working state recognition data set.

\begin{tabular}{lcc}
\hline Data set & Training set & Incremental set \\
\hline A & 320 & 80 \\
B & 320 & 80 \\
APAR & 480 & 120 \\
\hline
\end{tabular}

TABLE 8: Division of unknown working state recognition data set.

(a)

\begin{tabular}{lccc}
\hline Data set & $\begin{array}{c}\text { Training set } \\
(\text { S1-S3 })\end{array}$ & $\begin{array}{c}\text { Incremental set } \\
(\text { S4 })\end{array}$ & $\begin{array}{c}\text { Test set } \\
(\text { S1-S4) }\end{array}$ \\
\hline A & 240 & 80 & 80 \\
B & 240 & 80 & 80 \\
\hline
\end{tabular}

(b)

\begin{tabular}{lccc}
\hline Data set & $\begin{array}{c}\text { Training set } \\
(\text { S1-S5) }\end{array}$ & $\begin{array}{c}\text { Incremental set } \\
(\text { S6 })\end{array}$ & $\begin{array}{c}\text { Test set } \\
(\text { S1-S6) }\end{array}$ \\
\hline APAR & 400 & 80 & 120 \\
\hline
\end{tabular}

-means algorithm depends on the number of clusters and the selection of the initial clustering center. It is also sensitive to "noise" and "outliers." Because the iterative method often converges to a local minimum, the clustering effect is not ideal. The AP algorithm overcomes the disadvantage of the $K$-means algorithm. The algorithm does not need to specify the number of clusters and cluster centers and is not sensitive to the initial value of the data. However, the clustering result is still affected by parameters. The IAP algorithm firstly takes the clustering result of the PSO-AP algorithm as the initial clustering center, which overcomes the influence of the AP algorithm parameter values. Then, introduce the idea of the KNN algorithm to increment clustering,
TABLE 9: Parameter settings of $K$-means algorithm.

\begin{tabular}{lc}
\hline Data set & Clustering number $k$ \\
\hline A & 4 \\
B & 4 \\
APAR & 6 \\
\hline
\end{tabular}

which has a high tolerance for outliers and noise. Therefore, the IAP algorithm can achieve a better clustering effect in most cases. When appropriate parameters are selected, the IAP algorithm has the highest clustering purity, the AP algorithm is the second, and the $K$-means algorithm is the worst. For the IAP algorithm, the RI is lower than that of the $K$ -means algorithm only on the unknown working state clustering of A and B data sets, the RI is lower than that of the AP algorithm and $K$-means algorithm on the unknown working state clustering of APAR data sets, and the $F$-measure is lower than that of the $K$-means algorithm on the known working state clustering of the B data set. The IAP algorithm can always obtain good clustering results due to the proper and fixed initial center point

(2) From the perspective of time, the $K$-means algorithm is simple and efficient with the fastest convergence rate and only needs to calculate the distance between $N$ data points and $C$ centers. The time complexity is $O($ $N * C)(N$ is the number of samples; $C$ is the number of clustering). Due to the need to calculate the similarity between all sample points, the AP algorithm has a slow convergence speed and time complexity is $O$ $\left(N^{2} * \log N\right)$. When the sample size is large, the AP algorithm has low efficiency. Based on the current AP clustering results, the IAP algorithm uses the KNN idea to cluster incremental samples. Because the KNN algorithm is online technology, incremental samples can be directly added to the data set without retraining, and the algorithm's running time is greatly shortened. The time complexity is $O\left(M^{2} * \log M+I\right)$ ( $M<N, M$ is the number of samples, and $I$ is the number of incremental samples). The AP algorithm's efficiency is improved, especially for large-scale data, and the clustering speed is obvious

In summary, the $K$-means algorithm runs efficiently, but the clustering accuracy rate is low. The AP algorithm has a better clustering effect, but the algorithm runs inefficiently for incremental clustering. The IAP algorithm overcomes the shortcomings of the AP algorithm and $K$-means algorithm, improves the clustering accuracy and convergence speed, and proves the effectiveness and feasibility of IAP clustering in radar known and unknown working state recognition.

(3) Validity Analysis of the BP Recognition Model.

(a) Model parameter setting 
TABLE 10: Experimental results of algorithm comparison.

\begin{tabular}{lccccccccc}
\hline \multirow{2}{*}{ Data set } & \multirow{2}{*}{ Algorithm } & \multicolumn{2}{c}{ Purity } & \multicolumn{3}{c}{ RI } & \multicolumn{2}{c}{ F-measure } & \multicolumn{2}{c}{ Time (s) } \\
& & Known & Unknown & Known & Unknown & Known & Unknown & Known & Unknown \\
\hline \multirow{3}{*}{ A } & IAP & 0.821 & 0.863 & 0.847 & 0.760 & 0.594 & 0.926 & 33.18 & 26.10 \\
& AP & 0.733 & 0.797 & 0.800 & 0.725 & 0.538 & 0.798 & 51.64 & 48.11 \\
& K-means & 0.633 & 0.65 & 0.834 & 0.803 & 0.556 & 0.757 & 0.12 & 0.12 \\
\hline \multirow{2}{*}{ B } & IAP & 0.754 & 0.713 & 0.800 & 0.535 & 0.433 & 0.832 & 26.98 & 11.47 \\
& AP & 0.649 & 0.638 & 0.778 & 0.501 & 0.412 & 0.624 & 46.94 & 25.12 \\
& K-means & 0.473 & 0.516 & 0.680 & 0.675 & 0.551 & 0.542 & 0.13 & 0.12 \\
\hline \multirow{2}{*}{ APAR } & IAP & 0.919 & 0.813 & 0.951 & 0.669 & 0.846 & 0.897 & 66.56 & 42.80 \\
& AP & 0.845 & 0.773 & 0.917 & 0.711 & 0.775 & 0.872 & 102.07 & 63.31 \\
& K-means & 0.740 & 0.690 & 0.870 & 0.862 & 0.756 & 0.737 & 0.15 & 0.14 \\
\hline
\end{tabular}

The input layer and output layer node numbers are, respectively, determined by the dimension of the input feature vector and the desired output dimensions. The hidden layer node number is determined by the empirical formula and trial and error method. Assuming that the output result of corresponding each state is represented by numbers 1,2 , etc., the recognition model network structure parameters of three radars are set as shown in Table 11.

Other parameters were set as follows:

(i) Activation function of output layer: Purelin

(ii) Training function of neural network: Trainlm

(iii) Maximum iteration step of neural network: 1000

(iv) Training target error of neural network: 0.001

(v) Learning rate factor of neural network: 0.01

(b) Result analysis

Samples generated by IAP clustering of the three data sets in Section (2) of 4.3.3 were, respectively, input into BP neural network as training sets for model training. After the training is completed, the test set samples in Table 8 were input. Assuming that $\mathrm{S} 1-\mathrm{S} 3$ of $\mathrm{A}$ and $\mathrm{B}$ data sets are known states, S4 are unknown states, S1-S5 of APAR data sets are known states, and S6 are unknown states. The recognition results are shown in Figures 12-14.

As can be seen from the confusion matrix, the radar working state recognition model based on IAP clustering and BP neural network has a self-learning function, which correctly identifies the known and unknown working state. Because the improved AP algorithm uses the PSO algorithm to overcome the influence of the parameter value of the algorithm itself and introduces the KNN algorithm idea to divide the incremental samples, the accuracy of clustering is improved. The initial samples prelabeled by improved AP clustering are input into the BP neural network model for training. Using the good classification ability of the BP neural network, the recognition of radar working status is well completed. The
TABLE 11: BP network structure parameter settings.

\begin{tabular}{lccc}
\hline $\begin{array}{l}\text { Recognition } \\
\text { model }\end{array}$ & $\begin{array}{c}\text { Input layer } \\
\text { node number }\end{array}$ & $\begin{array}{c}\text { Hidden layer } \\
\text { node number }\end{array}$ & $\begin{array}{c}\text { Output layer } \\
\text { node number }\end{array}$ \\
\hline A & 3 & 7 & 1 \\
B & 3 & 7 & 1 \\
APAR & 5 & 8 & 1 \\
\hline
\end{tabular}

Predict state

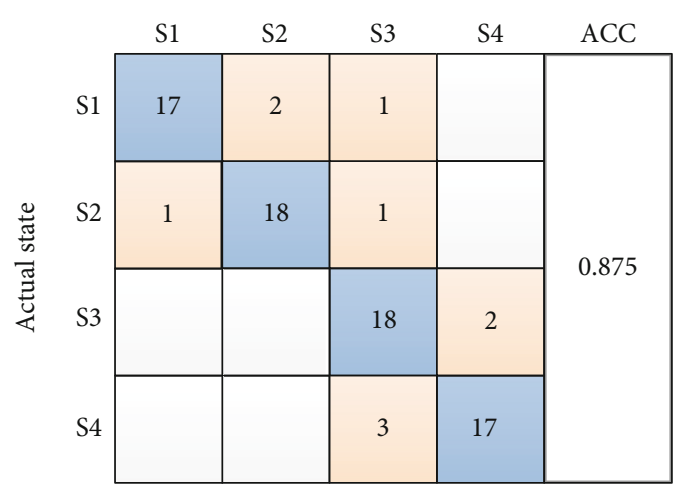

Figure 12: A data set prediction state confusion matrix.

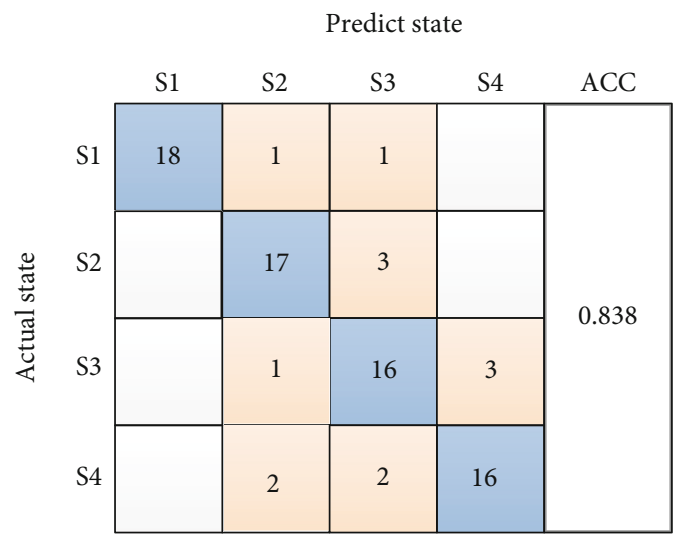

FIGURE 13: B data set prediction state confusion matrix. 


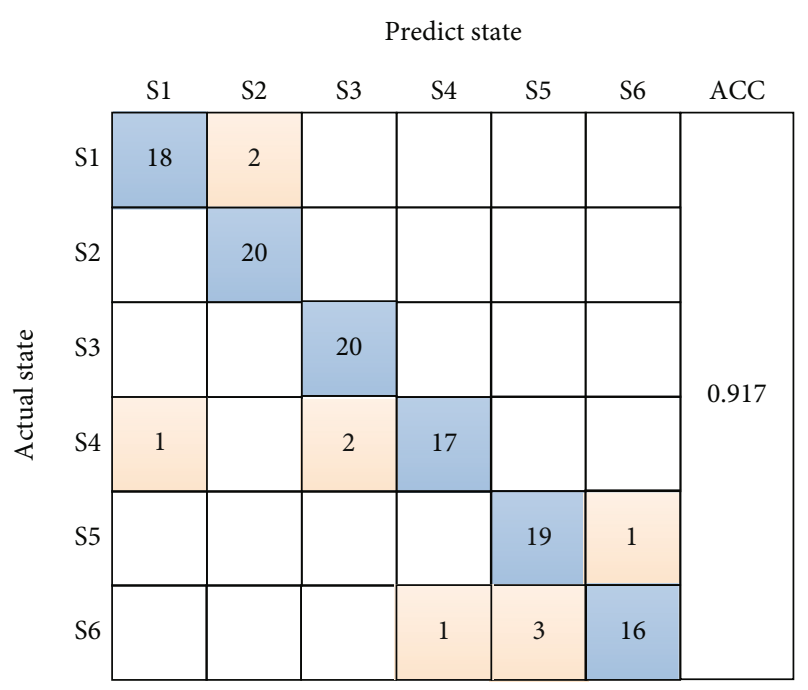

FIGURE 14: APAR data set prediction state confusion matrix.

overall recognition accuracy of the model for the three data sets is high, that is, respectively, $0.875,0.838$, and 0.917 .

\section{Conclusions}

For the traditional supervised working state recognition methods, the recognition efficiency is low due to the lack of timeliness and adaptability to the environment. This paper proposes an unsupervised incremental radar working state recognition model to quickly and accurately identify the category to which the state belongs and determine whether there is a new category when facing the continuous incoming signal data stream. At the same time, it can timely update existing classification models to adapt to changes in the external environment. The conclusions are as follows:

(1) Adopting the idea of "first cluster and then sort," the incremental PSO-AP algorithm and BP classification algorithm are combined to build an unsupervised and incremental radar working state recognition model and complete known state recognition and unknown state detection. The efficiency of the cognitive electronic countermeasure system can be further improved

(2) The improved AP algorithm can meet the prelabeling of the unsupervised initial training set and ensure the incremental recognition requirement. It automatically adjusts the parameters of the AP algorithm through the parameter optimization ability of the PSO algorithm, which improves the clustering effect. Moreover, the idea of KNN is introduced to realize IAP clustering. When processing incremental data, it avoids the traversal of all data and improves the clustering speed

(3) This model overcomes the disadvantages of the low efficiency of the traditional AP algorithm for incremental data clustering and low accuracy of the $K$ -means algorithm and has higher accuracy and faster convergence speed for different radar state clustering. The training samples obtained by the improved AP clustering make the BP model have a higher recognition rate

However, this paper also has some shortcomings. For example, the incremental processing of the IAP algorithm is carried out one by one, and when the incremental data is too large, the algorithm's efficiency may be greatly reduced. Therefore, we will study the incremental algorithm of batch processing in the future to further improve the efficiency of radar working state recognition.

\section{Data Availability}

The data used to support the findings of this study are included within the article.

\section{Conflicts of Interest}

The authors declare that they have no conflicts of interest.

\section{Acknowledgments}

This study was supported by the National Natural Science Foundation of China (61773389), Natural Science Foundation of Shaanxi Province (2020JQ-298), and Postdoctoral Science Foundation of China (2019M663635).

\section{References}

[1] J. Knowles, "Regaining the advantage-cognitive electronic warfare," The Journal of Electronic Defense, vol. 39, no. 12, pp. 5662, 2016.

[2] Z. Xiao and Z. Yan, "Radar emitter identification based on naive Bayesian algorithm," in Proceedings of the IEEE 5th Information Technology and Mechatronics Engineering Conference, pp. 1242-1244, Chongqing, China, 2020.

[3] R. Guillaume, M. Ali, and E. Cyrille, "Radar emitters classification and clustering with a scale mixture of normal distributions," in Proceedings of the IEEE Radar Conference, pp. 1371-1376, Oklahoma City, OK, USA, 2018.

[4] Y. N. Wei, Y. Li, and J. D. Zhang, "Radar jamming recognition method based on fuzzy clustering decision tree," in Proceedings of the IEEE International Conference on Signal, Information and Data Processing, pp. 1-5, Chongqing, China, 2019.

[5] S. Wang, J. Li, Y. Wang, and Y. Li, "Radar HRRP target recognition based on gradient boosting decision tree," in Proceedings of the 9th International Congress on Image and Signal Processing, BioMedical Engineering and Informatics, pp. 1013-1017, Datong, China, 2016.

[6] W. Gongming, C. Shiwen, H. Xueruobai, and Y. Junjian, "Radar emitter sorting and recognition based on timefrequency image union feature," in Proceedings of the IEEE 4th International Conference on Signal and Image Processing, pp. 165-170, Wuxi, China, 2019.

[7] X. Y. Wang, C. Su, and S. L. Sun, "An improved method of radar emitter fingerprint recognition based on GS-SVM," in Proceedings of the 19th International Symposium on Communications and Information Technologies, pp. 244-248, Ho Chi Minh City, Vietnam, 2019. 
[8] L. D. Ding, S. L. Wang, F. G. Wang, and W. Zhang, "Specific emitter identification via convolutional neural networks," IEEE Communications Letters, vol. 22, no. 12, pp. 2591-2594, 2018.

[9] J. Matuszewski, "Radar signal identification using a neural network and pattern recognition methods," in Proceedings of the 14th International Conference on Advanced Trends in Radioelecrtronics, Telecommunications and Computer Engineering, pp. 79-83, Lviv-Slavske, Ukraine, 2018.

[10] J. Matuszewski and K. Sikorska-Lukasiewicz, "Neural network application for emitter recognition," in Proceedings of the 18th International Radar Symposium, pp. 1-8, Prague, Czech Republic, 2017.

[11] H. Q. Li, Y. L. Li, X. M. Wang, Z. Xu, and X. Yin, "Radar working state recognition based on improved HPSO-BP," International Journal of Antennas and Propagation, vol. 2021, Article ID 5586851, 13 pages, 2021.

[12] M. W. Li, M. H. He, J. Han, and Y. W. Tang, "A new clustering and sorting algorithm for radar emitter signals," Journal of Physics: Conference Series, vol. 1617, no. 1, pp. 012009012010, 2020.

[13] X. Feng, X. X. Hu, and Y. Liu, "Radar signal sorting algorithm of k-means clustering based on data field," in Proceedings of the 3rd IEEE International Conference on Computer and Communications, pp. 2262-2266, Chengdu, China, 2017.

[14] Y. Liu and H. Y. Cui, "A novel self-adaptive density-based clustering algorithm for radar signal sorting," in Proceedings of the IET International Radar Conference, pp. 1-7, Hangzhou, China, 2015.

[15] K. Chi, J. H. Shen, Y. Li, Y. Li, and S. Wang, "Multi-function radar signal sorting based on complex network," IEEE Signal Processing Letters, vol. 28, pp. 91-95, 2021.

[16] M. G. S. Ahmed and B. Tang, "Sorting radar signal from symmetry clustering perspective," Journal of Systems Engineering and Electronics, vol. 28, no. 4, pp. 690-696, 2017.

[17] S. Cao, S. C. Wang, and Y. Zhang, "Density-based fuzzy Cmeans multi-center re-clustering radar signal sorting algorithm," in Proceedings of the 17th IEEE International Conference on Machine Learning and Applications, pp. 891-896, Orlando, FL, USA, 2018.

[18] B. J. Frey and D. Dueck, "Clustering by passing messages between data points," Science, vol. 315 , no. 5814, pp. 972976, 2007.

[19] J. S. Hu, H. L. Liu, and Z. Yan, “Adaptive affinity propagation algorithm based on new strategy of dynamic damping factor and preference," IEEJ Transactions on Electrical and Electronic Engineering, vol. 14, no. 1, pp. 97-104, 2019.

[20] L. L. Sun and C. H. Guo, "Incremental affinity propagation clustering based on message passing," IEEE Transactions on Knowledge and Data Engineering, vol. 26, no. 11, pp. 27312744, 2014.

[21] Y. Peng, J. F. Cheng, and R. X. Jiang, "Inversion of UEP signatures induced by ships based on PSO method," Defence Technology, vol. 16, no. 1, pp. 172-177, 2020.

[22] Z. Li, Radar Radiation Source and Working State Recognition, Xidian University, Xi'an, China, 2019.

[23] S. Y. Cui, Research on Target State Recognition Technology in Cognitive Electronic Warfare, Beijing Institute of Technology, Beijing, China, 2016.
[24] X. F. Song, Y. Zhang, Y. N. Guo, X. Y. Sun, and Y. L. Wang, "Variable-size cooperative coevolutionary particle swarm optimization for feature selection on high-dimensional data," IEEE Transactions on Evolutionary Computation, vol. 24, no. 5, pp. 882-895, 2020.

[25] Y. Hu, Y. Zhang, and D. W. Gong, "Multiobjective particle swarm optimization for feature selection with fuzzy cost," IEEE Transactions on Cybernetics, vol. 51, no. 2, pp. 874$888,2021$.

[26] Y. Xue, B. Xue, and M. J. Zhang, "Self-adaptive particle swarm optimization for large-scale feature selection in classification," ACM Transactions on Knowledge Discovery from Data, vol. 13, no. 5, pp. 1-27, 2019.

[27] X.-h. Wang, X.-p. Zhang, C.-x. Zhuang, Z.-n. Chen, and Z. Qin, "Automatically determining the number of affinity propagation clustering using particle swarm," in Proceedings of the 5th IEEE Conference on Industrial Electronics and Applications, pp. 1526-1530, Taichung, Taiwan, 2010.

[28] X. F. Song, Y. Zhang, D. W. Gong, and X. Z. Gao, “A fast hybrid feature selection based on correlation-guided clustering and particle swarm optimization for high-dimensional data," IEEE Transactions on Cybernetics, pp. 1-14, 2021.

[29] T. L. Tang, S. Y. Chen, M. Zhao, W. Huang, and J. Luo, "Very large-scale data classification based on K-means clustering and multi-kernel SVM," Soft Computing, vol. 23, no. 11, pp. 3793$3801,2019$. 